\title{
New Unified Matrix Upper Bound on the Solution of the Continuous Coupled Algebraic Riccati Equation
}

\author{
Jianhong $\mathrm{Xu}^{*}$ and Prasanthan Rajasingam ${ }^{\dagger}$ \\ Department of Mathematics \\ Southern Illinois University Carbondale \\ Carbondale, Illinois 62901, U.S.A.
}

December 3, 2014

\begin{abstract}
In this paper, a unified matrix upper solution bound is established for the continuous coupled algebraic Riccati equation arising from the optimal control of a Markovian jump linear system. In particular, the issue of rank deficiency with the control matrices is addressed because it may cause problems to some existing matrix upper solution bounds. The results of this paper not only apply to this rank deficient case but also contain those existing results as special cases.
\end{abstract}

Keywords: coupled algebraic Riccati equation, matrix solution bound, rank deficient, stabilizability, iterative algorithm, positive semidefinite, eigenvalue, $M$-matrix

AMS Subject Classification: 15A18, 15A39, 15A42, 15B57, 65F30, 93B05, $93 \mathrm{C} 05$

${ }^{*}$ Corresponding author. E-mail: jhxu@siu.edu, Phone: +1 (618) 453-6510, Fax: +1 (618) 453-5300. The research of this author is partially supported by NSF grant DMS1419028.

${ }^{\dagger} \mathrm{E}$-mail: prasanthanr@siu.edu 


\section{Introduction}

Assume $s, n \geq 2$. Let $S=\{1, \ldots, s\}, A_{i} \in \mathbb{R}^{n \times n}, Q_{i} \in \mathbb{R}^{n \times n}$ with $Q_{i}$ being (symmetric) positive semidefinite, $B_{i} \in \mathbb{R}^{n \times m}$, where $m \leq n$, and $\delta_{i, j} \geq 0$ such that $\sum_{j \in S \backslash\{i\}} \delta_{i, j}>0$. Then, the continuous coupled algebraic Riccati equation can be formulated as

$$
A_{i}^{T} P_{i}+P_{i} A_{i}-P_{i} B_{i} B_{i}^{T} P_{i}+\sum_{j \in S \backslash\{i\}} \delta_{i, j} P_{j}=-Q_{i}, \quad \forall i \in S .
$$

This equation arises from the optimal control of the Markovian jump linear system

$$
\frac{d}{d t} x(t)=A(r(t)) x(t)+B(r(t)) u(t),
$$

where $r(t)$ is a Markovian jump process, $A(r(t)) \in \mathbb{R}^{n \times n}, B(r(t)) \in \mathbb{R}^{n \times m}$, and $x(t) \in \mathbb{R}^{n}$ and $u(t) \in \mathbb{R}^{m}$ are the state and control vectors, respectively. For background material on the Markovian jump linear system and the role equation (1) plays, see [20]. The matrices $B_{i}$ in (1) are referred to as control matrices.

We assume herein that equation (1) has a positive semidefinite solution $P_{i} \in \mathbb{R}^{n \times n}$, where $i \in S$. For necessary and sufficient conditions on the existence and uniqueness of such a solution, see, for example, $[1,5,12]$. As a part of these conditions, it is often assumed in literature [1] that each pair $\left(A_{i}, B_{i}\right)$ is stabilizable, i.e. there exists some feedback matrix $K_{i} \in \mathbb{R}^{m \times n}$ such that all the eigenvalues of $A_{i}-B_{i} K_{i}$ have negative real parts. In fact, this stabilizability condition shall serve as a starting point for this work.

We explain, in passing, some notation being used throughout this paper. Given a (symmetric) matrix $A \in \mathbb{R}^{n \times n}$, we denote $A \succ 0(A \succeq 0$, resp.) if $A$ is positive definite (semidefinite, resp.) and, similarly, $A \prec 0$ ( $A \preceq 0$, resp.) if $A$ is negative definite (semidefinite, resp.). For any $A, B \in \mathbb{R}^{n \times n}, A \succ B$ ( $A \succeq B$, resp.) is equivalent to $A-B \succ 0(A-B \succeq 0$, resp.). The relations $A \prec B$ and $A \preceq B$ are interpreted in a similar way.

Recently, the problem of estimating bounds for the solution of the continuous coupled algebraic Riccati equation in (1) has drawn much attention $[6,7,8,9,10,22,23]$. Among these works, in particular, a unified, improved 
matrix solution bound was proposed in [22], which includes and strengthens the results in [8]. In a follow-up work [23], an iterative refinement algorithm was fully established, which further improves the results in $[8,22]$.

For motivations behind the research on matrix solution bounds, we refer the reader to the introductory sections in $[7,8]$. Such bounds turn out to be useful when it comes to time-delay system controller design, stability analysis, robust stabilization, and convergence of numerical algorithms. For the numerical solution of equation (1), for example, matrix solution bounds can be used to establish important monotone-type convergence results [16].

The results in $[8,22,23]$, however, hinges on a key assumption regarding the matrices $A_{i}$ and $B_{i}$, namely, for each $i$, there must exist some constant $\alpha_{i}>0$ such that

$$
A_{i}^{T}+A_{i} \prec 2 \alpha_{i} B_{i} B_{i}^{T} .
$$

Condition (2) can always be satisfied by choosing sufficiently large $\alpha_{i}$, provided that the control matrices $B_{i}$ satisfy $\operatorname{rank}\left(B_{i}\right)=n$. If $\operatorname{rank}\left(B_{i}\right)<n$ for some $i^{1}$, however, condition (2) could possibly fail with no viable values of $\alpha_{i}$ as shown by the following example [9, 24].

Suppose that, for some $i$,

$$
A_{i}=\left[\begin{array}{cc}
1 & 3 \\
0 & -7
\end{array}\right] \text { and } B_{i}=\left[\begin{array}{c}
0 \\
\sqrt{6}
\end{array}\right] \text {. }
$$

Then, one of the eigenvalues of

$$
A_{i}^{T}+A_{i}-2 \alpha_{i} B_{i} B_{i}^{T}=\left[\begin{array}{cc}
2 & 3 \\
3 & -14-12 \alpha_{i}
\end{array}\right]
$$

is always positive for any $\alpha_{i}>0$, leading to the failure of condition (2). We point out that, as a matter of fact, rank deficiency with control matrices is often encountered in applications.

The primary goal of this paper is to address the issue of possible failure of condition (2) when it comes to deriving an upper bound for the solution

\footnotetext{
${ }^{1}$ This means that $B_{i} B_{i}^{T}$ is rank deficient. For convenience, we shall loosely refer to this as rank deficiency with the control matrices.
} 
of the continuous coupled algebraic Riccati equation in (1).

The main thrust for this study came from [9], which, nevertheless, dealt exclusively with the non-coupled situation involving a single continuous algebraic Riccati equation. Specifically, as in [9], we shall replace condition (2) with the more feasible stabilizability condition on each pair $\left(A_{i}, B_{i}\right)$. Under this new condition, together with the unified framework in $[22,23]$, we shall develop a new matrix upper bound on the solution of equation (1).

Our main contributions in this work may be summarized as follows.

(i) We show that, combined with the unifying methodology in [22] involving some $M$-matrix inequality, the techniques of [9] can be successfully extended to deal with the coupled case of continuous algebraic Riccati equations.

(ii) We generalize the results of $[22,23]$ with the usual stabilizability condition, which is less restrictive compared with condition (2).

(iii) Our results turn out to be broader as they contain the existing results in $[8,9,22,23]$ as special cases. This is indeed the reason why we call the new matrix upper bound here, again, a unified bound.

(iv) Because of their generality, an additional benefit of the unified results here is that they can be exploited to verify and obtain tighter solution bounds. We shall be able to show that, for instance, the bound in $[9$, Theorem 2.2] is always sharper than that in [9, Theorem 2.1].

\section{New Unified Matrix Upper Bound}

We quote first a number of necessary known results from the literature. In what follows, for a symmetric matrix $A \in \mathbb{R}^{n \times n}$, we denote by $\lambda_{1}(A)$ and $\lambda_{n}(A)$ its largest and smallest eigenvalues, respectively; for any rectangular matrix $A=\left[a_{i, j}\right]$, we denote $A \geq 0(A>0$, resp. $)$ if $a_{i, j} \geq 0\left(a_{i, j}>0\right.$, resp. $)$ for all $i$ and $j$.

LEMMA 2.1 ([14, p.96]) Given $A \in \mathbb{R}^{n \times n}$, all the eigenvalues of $A$ have negative real parts if and only if there exists a positive definite matrix $H \in$ $\mathbb{R}^{n \times n}$ such that

$$
A^{T} H+H A \prec 0 .
$$


We mention that Lemma 2.1 is the well-known Lyapunov's theorem. By convention, such a matrix $A$ whose eigenvalues have negative real parts is said to be Hurwitz stable or (c-)stable; see, for example, [17, p.90].

The next result follows from Sylvester's law of inertia, which says that the relations such as $\prec$ are preserved under nonsingular congruences.

LEMMA 2.2 ([13, p.470]) Let $A, B \in \mathbb{R}^{n \times n}$ be symmetric such that $A \preceq B$ ( $A \prec B$, resp.). Then, for any nonsingular $C \in \mathbb{R}^{n \times n}$,

$$
C^{T} A C \preceq C^{T} B C \quad\left(C^{T} A C \prec C^{T} B C \text { resp. }\right) .
$$

We shall also need the next three technical lemmas from [22].

LEMMA 2.3 ([22, Lemma 3.1]) Let $C \in \mathbb{R}^{n \times n}$ be such that

$$
C^{T}+C \prec 0 .
$$

Then, for any $\gamma<0, C+\gamma I$ is nonsingular.

LEMMA 2.4 ([22, Lemma 3.2]) Suppose that $C \in \mathbb{R}^{n \times n}$ satisfies (3). Then, for any $\gamma<0$ and $\beta>\gamma$,

$$
\lambda_{1}\left\{(C+\gamma I)^{-T}\left[(C+\beta I)^{T}(C+\beta I)-\left(\beta^{2}-\gamma^{2}\right) I\right](C+\gamma I)^{-1}\right\}<1,
$$

where, and in the rest of this paper, the superscript -T means matrix inverse plus transpose.

LEMMA $2.5([22,(12) \&(15)])$ Assume that $C \in \mathbb{R}^{n \times n}$ satisfies (3). Then for any $\gamma<0$ and $\beta>\gamma$,

$$
\begin{aligned}
& 1-\lambda_{1}\left\{(C+\gamma I)^{-T}\left[(C+\beta I)^{T}(C+\beta I)-\left(\beta^{2}-\gamma^{2}\right) I\right](C+\gamma I)^{-1}\right\} \\
& =-(\beta-\gamma) \lambda_{1}\left[(C+\gamma I)^{-T}\left(C^{T}+C\right)(C+\gamma I)^{-1}\right] .
\end{aligned}
$$

In addition, we shall use the following two results, where the first one is due to H. Weyl.

LEMMA 2.6 ([3, Theorems 8.4.9\& 8.4.11]) For any symmetric matrices $A, B \in \mathbb{R}^{n \times n}$,

$$
\lambda_{1}(A)+\lambda_{n}(B) \leq \lambda_{1}(A+B) \leq \lambda_{1}(A)+\lambda_{1}(B) ;
$$

moreover, $\lambda_{1}(A) \leq \lambda_{1}(B)$ when $A \preceq B$. 
LEMMA 2.7 ([3, Corollary 8.4.2]) For any symmetric matrix $A \in \mathbb{R}^{n \times n}$,

$$
A \preceq \lambda_{1}(A) I .
$$

Recall now that $A=\left[a_{i, j}\right] \in \mathbb{R}^{n \times n}$ is said to be a $Z$-matrix and denoted as $A \in \mathbb{Z}^{n \times n}$ if $a_{i, j} \leq 0$ whenever $i \neq j$. If $A \in \mathbb{Z}^{n \times n}$ is nonsingular with $A^{-1} \geq 0$, then $A$ is called a (nonsingular) $M$-matrix.

LEMMA 2.8 ([4, p.137]) Suppose that $A \in \mathbb{Z}^{n \times n}$. Then $A$ is an M-matrix if and only if the following two conditions hold:

(i) A has all positive diagonal entries.

(ii) There exists a diagonal matrix $D$ whose diagonal entries are all positive such that $A D$ is strictly diagonally dominant.

From now on, for the continuous coupled algebraic Riccati equation (1), we shall always assume that each pair $\left(A_{i}, B_{i}\right)$ is stabilizable, namely there exists $K_{i} \in \mathbb{R}^{m \times n}$ such that $A_{i}-B_{i} K_{i}$ is stable. Due to this stabilizability condition and Lemma 2.1, we know that for each $i \in S$, there exists positive definite $H_{i} \in \mathbb{R}^{n \times n}$ that satisfies

$$
C_{i}^{T} H_{i}+H_{i} C_{i} \prec 0 .
$$

where $C_{i}=A_{i}-B_{i} K_{i}$. We point out here that there are efficient algorithms for finding the matrices $K_{i}$, see [21] and the references therein.

We write $H_{i}=M_{i}^{T} M_{i}$ with $M_{i} \in \mathbb{R}^{n \times n}$ being nonsingular and let $\widetilde{C}_{i}=$ $M_{i} C_{i} M_{i}^{-1}$. Clearly, (4) is equivalent to

$$
\widetilde{C}_{i}^{T}+\widetilde{C}_{i} \prec 0
$$

according to Lemma 2.2 .

Following [22, 23], we shall also assume in the sequel that the constants $\beta_{i}$ and $\gamma_{i}$ are such that

$$
\gamma_{i}<0 \text { and } \gamma_{i}<\beta_{i} \leq-\gamma_{i}, \forall i \in S .
$$

We define now $F=\left[f_{i, j}\right] \in \mathbb{R}^{s \times s}$ and $g=\left[g_{1}, \ldots, g_{s}\right]^{T} \in \mathbb{R}^{s}$ as follows: 


$$
f_{i, j}=\left\{\begin{array}{cc}
1, & i=j \\
\frac{\delta_{i, j} \lambda_{1}\left[\left(\widetilde{C}_{i}+\gamma_{i} I\right)^{-T} M_{i}^{-T} M_{j}^{T} M_{j} M_{i}^{-1}\left(\widetilde{C}_{i}+\gamma_{i} I\right)^{-1}\right]}{\lambda_{1}\left[\left(\widetilde{C}_{i}+\gamma_{i} I\right)^{-T}\left(\widetilde{C}_{i}^{T}+\widetilde{C}_{i}\right)\left(\widetilde{C}_{i}+\gamma_{i} I\right)^{-1}\right]}, & i \neq j
\end{array}\right.
$$

and

$$
g_{i}=-\frac{\lambda_{1}\left[\left(\widetilde{C}_{i}+\gamma_{i} I\right)^{-T} M_{i}^{-T}\left(Q_{i}+K_{i}^{T} K_{i}\right) M_{i}^{-1}\left(\widetilde{C}_{i}+\gamma_{i} I\right)^{-1}\right]}{\lambda_{1}\left[\left(\widetilde{C}_{i}+\gamma_{i} I\right)^{-T}\left(\widetilde{C}_{i}^{T}+\widetilde{C}_{i}\right)\left(\widetilde{C}_{i}+\gamma_{i} I\right)^{-1}\right]} .
$$

Observe that by (5) and Lemma 2.3, $F$ and $g$ are both well-defined.

THEOREM 2.1 Suppose that for each $i,\left(A_{i}, B_{i}\right)$ is stabilizable. Let $H_{i}$ be given as in (4). Then we have $F \in \mathbb{Z}^{s \times s}$ and $g \geq 0$. Moreover, $F$ and $g$ can be written as

$$
f_{i, j}=\left\{\begin{array}{cc}
1, & i=j \\
\frac{\delta_{i, j} \lambda_{1}\left[\left(C_{i}+\gamma_{i} I\right)^{-1} H_{i}^{-1}\left(C_{i}+\gamma_{i} I\right)^{-T} H_{j}\right]}{\lambda_{1}\left[\left(C_{i}+\gamma_{i} I\right)^{-1} H_{i}^{-1}\left(C_{i}+\gamma_{i} I\right)^{-T}\left(C_{i}^{T} H_{i}+H_{i} C_{i}\right)\right]}, & i \neq j
\end{array}\right.
$$

and

$$
g_{i}=-\frac{\lambda_{1}\left[\left(C_{i}+\gamma_{i} I\right)^{-1} H_{i}^{-1}\left(C_{i}+\gamma_{i} I\right)^{-T}\left(Q_{i}+K_{i}^{T} K_{i}\right)\right]}{\lambda_{1}\left[\left(C_{i}+\gamma_{i} I\right)^{-1} H_{i}^{-1}\left(C_{i}+\gamma_{i} I\right)^{-T}\left(C_{i}^{T} H_{i}+H_{i} C_{i}\right)\right]} .
$$

If, in addition,

$$
\begin{gathered}
\lambda_{1}\left[\left(C_{i}+\gamma_{i} I\right)^{-1} H_{i}^{-1}\left(C_{i}+\gamma_{i} I\right)^{-T}\left(C_{i}^{T} H_{i}+H_{i} C_{i}\right)\right] \\
+\sum_{j \in S \backslash\{i\}} \delta_{i, j} \lambda_{1}\left[\left(C_{i}+\gamma_{i} I\right)^{-1} H_{i}^{-1}\left(C_{i}+\gamma_{i} I\right)^{-T} H_{j}\right]<0,
\end{gathered}
$$

then $F$ is an $M$-matrix. 
Proof: By condition (5) and Lemmas 2.2 and 2.6, we see immediately that $f_{i, j}<0$ for any $i \neq j$ and that $g_{i} \geq 0$ for any $i$. Next, formulas (8) and (9) follow directly from (6) and (7) by the fact that the eigenvalues of a matrix are invariant under similarity transformations. Finally, we notice that condition (10) implies that $F$ must be a strictly diagonally dominant matrix and thus, by Lemma $2.8, F$ is an $M$-matrix.

Observe that, compared with (6) and (7), formulas (8) and (9) above in Theorem 2.1 are more convenient since both are expressed directly in terms of $C_{i}$ and $H_{i}$ as in condition (4).

Under the stabilizability condition on $\left(A_{i}, B_{i}\right)$ and condition $(10)$, we set $\xi \in \mathbb{R}^{s}$ as the unique solution to the linear system

$$
F \xi=g
$$

We comment that, according to Theorem 2.1, $\xi \geq 0$. Moreover, we note here that $F$ and $g$ are both independent of $\beta_{i}$, and so is $\xi$.

We are now ready to formulate our new, unified matrix upper bound on the solution of the continuous coupled algebraic Riccati equation in (1).

THEOREM 2.2 Let $\gamma_{i}<0$ and $\gamma_{i}<\beta_{i} \leq-\gamma_{i}$ for all $i \in S$. Suppose that each pair $\left(A_{i}, B_{i}\right)$ is stabilizable, i.e. $C_{i}=A_{i}-B_{i} K_{i}$ is stable for some $K_{i}$, such that condition (4) holds with $H_{i}$ being positive definite. In addition, we suppose that condition (10) also holds. Then, we have

$$
\begin{aligned}
P_{i} \preceq & \left(C_{i}+\gamma_{i} I\right)^{-T}\left\{\xi_{i}\left[\left(C_{i}+\beta_{i} I\right)^{T} H_{i}\left(C_{i}+\beta_{i} I\right)-\left(\beta_{i}^{2}-\gamma_{i}^{2}\right) H_{i}\right]\right. \\
& \left.+\left(\beta_{i}-\gamma_{i}\right)\left(\sum_{j \in S \backslash\{i\}} \delta_{i, j} \xi_{j} H_{j}+Q_{i}+K_{i}^{T} K_{i}\right)\right\}\left(C_{i}+\gamma_{i} I\right)^{-1},
\end{aligned}
$$

where $\xi$ is given by (11).

Proof: Once again, we write that $H_{i}=M_{i}^{T} M_{i}$, where $M_{i} \in \mathbb{R}^{n \times n}$ is nonsingular. For convenience, set $\widetilde{A}_{i}=M_{i} A_{i} M_{i}^{-1}, \widetilde{B}_{i}=M_{i} B_{i}, \widetilde{Q}_{i}=M_{i}^{-T} Q_{i} M_{i}^{-1}$, and $\widetilde{P}_{i}=M_{i}^{-T} P_{i} M_{i}^{-1}$. Then (1) can be written as

$$
\widetilde{A}_{i}^{T} \widetilde{P}_{i}+\widetilde{P}_{i} \widetilde{A}_{i}-\widetilde{P}_{i} \widetilde{B}_{i} \widetilde{B}_{i}^{T} \widetilde{P}_{i}+\sum_{j \in S \backslash\{i\}} \delta_{i, j} M_{i}^{-T} M_{j}^{T} \widetilde{P}_{j} M_{j} M_{i}^{-1}+\widetilde{Q}_{i}=0 .
$$


Since

$$
\begin{aligned}
\widetilde{P}_{i} \widetilde{B}_{i} \widetilde{B}_{i}^{T} \widetilde{P}_{i}= & \left(\widetilde{B}_{i}^{T} \widetilde{P}_{i}-K_{i} M_{i}^{-1}\right)^{T}\left(\widetilde{B}_{i}^{T} \widetilde{P}_{i}-K_{i} M_{i}^{-1}\right)+M_{i}^{-T} K_{i}^{T} \widetilde{B}_{i}^{T} \widetilde{P}_{i} \\
& +\widetilde{P}_{i} \widetilde{B}_{i} K_{i} M_{i}^{-1}-M_{i}^{-T} K_{i}^{T} K_{i} M_{i}^{-1}
\end{aligned}
$$

and

$$
\left(\widetilde{B}_{i}^{T} \widetilde{P}_{i}-K_{i} M_{i}^{-1}\right)^{T}\left(\widetilde{B}_{i}^{T} \widetilde{P}_{i}-K_{i} M_{i}^{-1}\right) \succeq 0,
$$

we obtain from (13) that

$$
\widetilde{C}_{i}^{T} \widetilde{P}_{i}+\widetilde{P}_{i} \widetilde{C}_{i}+\sum_{j \in S \backslash\{i\}} \delta_{i, j} M_{i}^{-T} M_{j}^{T} \widetilde{P}_{j} M_{j} M_{i}^{-1}+\widetilde{Q}_{i}+M_{i}^{-T} K_{i}^{T} K_{i} M_{i}^{-1} \succeq 0,
$$

where $\widetilde{C}_{i}=\widetilde{A}_{i}-\widetilde{B}_{i} K_{i} M_{i}^{-1}$. Next, using

$$
\begin{aligned}
& \left(\beta_{i}-\gamma_{i}\right)\left(\widetilde{C}_{i}^{T} \widetilde{P}_{i}+\widetilde{P}_{i} \widetilde{C}_{i}\right) \\
& =\left(\widetilde{C}_{i}+\beta_{i} I\right)^{T} \widetilde{P}_{i}\left(\widetilde{C}_{i}+\beta_{i} I\right)-\left(\widetilde{C}_{i}+\gamma_{i} I\right)^{T} \widetilde{P}_{i}\left(\widetilde{C}_{i}+\gamma_{i} I\right)-\left(\beta_{i}^{2}-\gamma_{i}^{2}\right) \widetilde{P}_{i},
\end{aligned}
$$

(14), and Lemmas 2.2 and 2.3, we arrive at

$$
\begin{aligned}
& \widetilde{P}_{i} \preceq \\
& \left(\widetilde{C}_{i}+\gamma_{i} I\right)^{-T}\left[\left(\widetilde{C}_{i}+\beta_{i} I\right)^{T} \widetilde{P}_{i}\left(\widetilde{C}_{i}+\beta_{i} I\right)-\left(\beta_{i}^{2}-\gamma_{i}^{2}\right) \widetilde{P}_{i}\right. \\
& \left.+\left(\beta_{i}-\gamma_{i}\right) M_{i}^{-T}\left(\sum_{j \in S \backslash\{i\}} \delta_{i, j} M_{j}^{T} \widetilde{P}_{j} M_{j}+Q_{i}+K_{i}^{T} K_{i}\right) M_{i}^{-1}\right]\left(\widetilde{C}_{i}+\gamma_{i} I\right)^{-1} .
\end{aligned}
$$

By applying Lemma 2.7 and then Lemma 2.6, the above further reduces to

$$
\begin{aligned}
& \widetilde{P}_{i} \preceq \\
& \lambda_{1}\left(\widetilde{P}_{i}\right)\left(\widetilde{C}_{i}+\gamma_{i} I\right)^{-T}\left[\left(\widetilde{C}_{i}+\beta_{i} I\right)^{T}\left(\widetilde{C}_{i}+\beta_{i} I\right)-\left(\beta_{i}^{2}-\gamma_{i}^{2}\right) I\right]\left(\widetilde{C}_{i}+\gamma_{i} I\right)^{-1} \\
& +\left(\beta_{i}-\gamma_{i}\right) \sum_{j \in S \backslash\{i\}} \delta_{i, j} \lambda_{1}\left(\widetilde{P}_{j}\right)\left(\widetilde{C}_{i}+\gamma_{i} I\right)^{-T} M_{i}^{-T} M_{j}^{T} M_{j} M_{i}^{-1}\left(\widetilde{C}_{i}+\gamma_{i} I\right)^{-1} \\
& +\left(\beta_{i}-\gamma_{i}\right)\left(\widetilde{C}_{i}+\gamma_{i} I\right)^{-T} M_{i}^{-T}\left(Q_{i}+K_{i}^{T} K_{i}\right) M_{i}^{-1}\left(\widetilde{C}_{i}+\gamma_{i} I\right)^{-1}
\end{aligned}
$$


and then

$$
\begin{aligned}
& \lambda_{1}\left(\widetilde{P}_{i}\right) \leq \\
& \lambda_{1}\left(\widetilde{P}_{i}\right) \lambda_{1}\left\{\left(\widetilde{C}_{i}+\gamma_{i} I\right)^{-T}\left[\left(\widetilde{C}_{i}+\beta_{i} I\right)^{T}\left(\widetilde{C}_{i}+\beta_{i} I\right)-\left(\beta_{i}^{2}-\gamma_{i}^{2}\right) I\right]\left(\widetilde{C}_{i}+\gamma_{i} I\right)^{-1}\right\} \\
& +\left(\beta_{i}-\gamma_{i}\right) \sum_{j \in S \backslash\{i\}} \delta_{i, j} \lambda_{1}\left(\widetilde{P}_{j}\right) \lambda_{1}\left[\left(\widetilde{C}_{i}+\gamma_{i} I\right)^{-T} M_{i}^{-T} M_{j}^{T} M_{j} M_{i}^{-1}\left(\widetilde{C}_{i}+\gamma_{i} I\right)^{-1}\right] \\
& +\left(\beta_{i}-\gamma_{i}\right) \lambda_{1}\left[\left(\widetilde{C}_{i}+\gamma_{i} I\right)^{-T} M_{i}^{-T}\left(Q_{i}+K_{i}^{T} K_{i}\right) M_{i}^{-1}\left(\widetilde{C}_{i}+\gamma_{i} I\right)^{-1}\right], \quad \forall i \in S .
\end{aligned}
$$

Now, according to Lemmas 2.4 and 2.5, the last inequality turns out to be, in matrix form, simply

$$
F x \leq g,
$$

where $x=\left[\lambda_{1}\left(\widetilde{P}_{1}\right), \ldots, \lambda_{1}\left(\widetilde{P}_{s}\right)\right]^{T}$ and where $F$ and $g$ are as given in (6) and (7), respectively. This, noticing here that $F^{-1} \geq 0$ by Theorem 2.1 , leads to $x \leq F^{-1} g=\xi$, i.e.

$$
\lambda_{1}\left(\widetilde{P}_{i}\right) \leq \xi_{i}
$$

for each $i \in S$. This estimate of $\lambda_{1}\left(\widetilde{P}_{i}\right)$, together with (15) and noting there that $\beta_{i}^{2}-\gamma_{i}^{2} \leq 0$, produce the following matrix upper bound on $\widetilde{P}_{i}$ :

$$
\begin{aligned}
& \widetilde{P}_{i} \preceq \\
& \left(\widetilde{C}_{i}+\gamma_{i} I\right)^{-T}\left\{\xi_{i}\left[\left(\widetilde{C}_{i}+\beta_{i} I\right)^{T}\left(\widetilde{C}_{i}+\beta_{i} I\right)-\left(\beta_{i}^{2}-\gamma_{i}^{2}\right) I\right]\right. \\
& \left.+\left(\beta_{i}-\gamma_{i}\right) M_{i}^{-T}\left(\sum_{j \in S \backslash\{i\}} \delta_{i, j} \xi_{j} M_{j}^{T} M_{j}+Q_{i}+K_{i}^{T} K_{i}\right) M_{i}^{-1}\right\}\left(\widetilde{C}_{i}+\gamma_{i} I\right)^{-1} .
\end{aligned}
$$

Finally, the conclusion in (12) can be readily verified using this last expression, $\widetilde{C}_{i}=M_{i} C_{i} M_{i}^{-1}$, and $M_{i}^{T} M_{i}=H_{i}$.

We comment that, compared with existing results, Theorem 2.2 is more general. Specifically, we see that [22, Theorem 3.3], which includes results in [8], is now a particular case of Theorem 2.2 with $H_{i}=I$ and $K_{i}=\alpha_{i} B_{i}^{T}$, while [9, Theorems $1 \& 2$ ] are also consequences of Theorem 2.2 if we allow $s=1$. For this latter case, we observe that (15) simply reduces to $[9,(12)]$ when $\beta_{1}=0$ and $\gamma_{1}=-1$, and to $[9,(27)]$ when $\beta_{1}=1$ and $\gamma_{1}=-1$, 
respectively; furthermore, $\xi_{1}=g_{1}$, which turns out to be exactly the same as the quantity $\rho$ in $[9]$.

Before continuing, we need the following conclusion.

THEOREM 2.3 Let each pair $\left(A_{i}, B_{i}\right)$ be stabilizable, i.e. $C_{i}=A_{i}-B_{i} K_{i}$ be stable for some $K_{i}$ and $H_{i}$ be positive definite so that condition (4) holds. Then, condition (10) implies

$$
\xi_{i}\left(C_{i}^{T} H_{i}+H_{i} C_{i}\right)+\sum_{j \in S \backslash\{i\}} \delta_{i, j} \xi_{j} H_{j}+Q_{i}+K_{i}^{T} K_{i} \preceq 0
$$

where $\xi$ is determined by (11).

Proof: By Lemma 2.2, it suffices to show that the largest eigenvalue of

$$
\left(C_{i}+\gamma_{i} I\right)^{-T}\left[\xi_{i}\left(C_{i}^{T} H_{i}+H_{i} C_{i}\right)+\sum_{j \in S \backslash\{i\}} \delta_{i, j} \xi_{j} H_{j}+Q_{i}+K_{i}^{T} K_{i}\right]\left(C_{i}+\gamma_{i} I\right)^{-1}
$$

is less than or equal to zero. Returning to (11), we know that

$$
\xi_{i}+\sum_{j \in S \backslash\{i\}} f_{i, j} \xi_{j}=g_{i}, \forall i \in S
$$

i.e.

$$
\begin{aligned}
& \xi_{i} \lambda_{1}\left[\left(C_{i}+\gamma_{i} I\right)^{-1} H_{i}^{-1}\left(C_{i}+\gamma_{i} I\right)^{-T}\left(C_{i}^{T} H_{i}+H_{i} C_{i}\right)\right] \\
& +\sum_{j \in S \backslash\{i\}} \delta_{i, j} \xi_{j} \lambda_{1}\left[\left(C_{i}+\gamma_{i} I\right)^{-1} H_{i}^{-1}\left(C_{i}+\gamma_{i} I\right)^{-T} H_{j}\right] \\
& +\lambda_{1}\left[\left(C_{i}+\gamma_{i} I\right)^{-1} H_{i}^{-1}\left(C_{i}+\gamma_{i} I\right)^{-T}\left(Q_{i}+K_{i}^{T} K_{i}\right)\right]=0
\end{aligned}
$$

by observing (8) and (9). Next, using Lemma 2.6, we have

$$
\begin{aligned}
& \lambda_{1}\left[\xi_{i}\left(C_{i}+\gamma_{i} I\right)^{-1} H_{i}^{-1}\left(C_{i}+\gamma_{i} I\right)^{-T}\left(C_{i}^{T} H_{i}+H_{i} C_{i}\right)\right. \\
& +\sum_{j \in S \backslash\{i\}} \delta_{i, j} \xi_{j}\left(C_{i}+\gamma_{i} I\right)^{-1} H_{i}^{-1}\left(C_{i}+\gamma_{i} I\right)^{-T} H_{j} \\
& \left.+\left(C_{i}+\gamma_{i} I\right)^{-1} H_{i}^{-1}\left(C_{i}+\gamma_{i} I\right)^{-T}\left(Q_{i}+K_{i}^{T} K_{i}\right)\right] \leq 0 .
\end{aligned}
$$


Since matrix eigenvalues are invariant under similarity transformations, on letting $H_{i}^{-1}=M_{i}^{-1} M_{i}^{-T}$, the above is equivalent to

$$
\begin{aligned}
\lambda_{1}\left\{M_{i}^{-T}\left(C_{i}+\gamma_{i} I\right)^{-T}[\right. & \xi_{i}\left(C_{i}^{T} H_{i}+H_{i} C_{i}\right)+\sum_{j \in S \backslash\{i\}} \delta_{i, j} \xi_{j} H_{j} \\
& \left.\left.+Q_{i}+K_{i}^{T} K_{i}\right]\left(C_{i}+\gamma_{i} I\right)^{-1} M_{i}^{-1}\right\} \leq 0 .
\end{aligned}
$$

This, by Lemma 2.2, completes the proof.

Condition (16) allows us not only to obtain the following monotonicity result but also to justify, see Section 3, an iterative refinement algorithm for improving the matrix upper solution bound in Theorem 2.2.

THEOREM 2.4 Under the same conditions as in Theorem 2.2, we fix all $\gamma_{i}<0$ and let $\gamma_{i}<\beta_{i}^{(1)} \leq \beta_{i}^{(2)} \leq-\gamma_{i}$. Let

$$
\begin{aligned}
U_{i}\left(\beta_{i}\right)= & \left(C_{i}+\gamma_{i} I\right)^{-T}\left\{\xi_{i}\left[\left(C_{i}+\beta_{i} I\right)^{T} H_{i}\left(C_{i}+\beta_{i} I\right)-\left(\beta_{i}^{2}-\gamma_{i}^{2}\right) H_{i}\right]\right. \\
& \left.+\left(\beta_{i}-\gamma_{i}\right)\left(\sum_{j \in S \backslash\{i\}} \delta_{i, j} \xi_{j} H_{j}+Q_{i}+K_{i}^{T} K_{i}\right)\right\}\left(C_{i}+\gamma_{i} I\right)^{-1} .
\end{aligned}
$$

Then

$$
U_{i}\left(\beta_{i}^{(2)}\right) \preceq U_{i}\left(\beta_{i}^{(1)}\right) .
$$

In particular, if $\beta_{i}^{(1)}<\beta_{i}^{(2)}$ and condition (16) holds with $\prec$, then the above holds with $\prec$.

Proof: For simplicity, we define here

$$
\begin{aligned}
\widehat{U}_{i}\left(\beta_{i}\right)= & \xi_{i}\left[\left(C_{i}+\beta_{i} I\right)^{T} H_{i}\left(C_{i}+\beta_{i} I\right)-\left(\beta_{i}^{2}-\gamma_{i}^{2}\right) H_{i}\right] \\
& +\left(\beta_{i}-\gamma_{i}\right)\left(\sum_{j \in S \backslash\{i\}} \delta_{i, j} \xi_{j} H_{j}+Q_{i}+K_{i}^{T} K_{i}\right) .
\end{aligned}
$$

Because of Lemma 2.2, we only need to show

$$
\widehat{U}_{i}\left(\beta_{i}^{(2)}\right) \preceq \widehat{U}_{i}\left(\beta_{i}^{(1)}\right) .
$$

By direct computation, we find that

$$
\begin{aligned}
& \widehat{U}_{i}\left(\beta_{i}^{(2)}\right)-\widehat{U}_{i}\left(\beta_{i}^{(1)}\right) \\
& =\left(\beta_{i}^{(2)}-\beta_{i}^{(1)}\right)\left[\xi_{i}\left(C_{i}^{T} H_{i}+H_{i} C_{i}\right)+\sum_{j \in S \backslash\{i\}} \delta_{i, j} \xi_{j} H_{j}+Q_{i}+K_{i}^{T} K_{i}\right] .
\end{aligned}
$$


The conclusions now follow easily from the above expression.

Theorem 2.4 shows that if $\gamma_{i}$ are all fixed, then the best possible matrix upper bound in the form of (12) is achieved when $\beta_{i}=-\gamma_{i}$. Hence, we have the following corollary, whose proof is immediate from Theorem 2.4.

COROLLARY 2.1 Under the same assumptions of Theorem 2.2, the following matrix upper solution bound is the tightest among all bounds in the form of (12) with fixed $\gamma_{i}<0$ and varying $\beta_{i}$ such that $\gamma_{i}<\beta_{i} \leq-\gamma_{i}$ :

$$
\begin{aligned}
P_{i} \preceq & \left(C_{i}+\gamma_{i} I\right)^{-T}\left[\xi_{i}\left(C_{i}-\gamma_{i} I\right)^{T} H_{i}\left(C_{i}-\gamma_{i} I\right)\right. \\
& \left.-2 \gamma_{i}\left(\sum_{j \in S \backslash\{i\}} \delta_{i, j} \xi_{j} H_{j}+Q_{i}+K_{i}^{T} K_{i}\right)\right]\left(C_{i}+\gamma_{i} I\right)^{-1} .
\end{aligned}
$$

As direct application of Corollary 2.1, we now see that [9, Theorem 2.1] always gives bounds that are tighter than those from [9, Theorem 2.1].

Returning to (4), we mention that we may choose $H_{i}$ such that

$$
C_{i}^{T} H_{i}+H_{i} C_{i}=-\rho_{i} I
$$

for some $\rho_{i}>0$. This choice was adopted in [9] for the non-coupled case of a single continuous algebraic Riccati equation. We proceed to show next an interesting feature of the matrix upper solution bound in (12), namely that it does not depend on $\rho_{i}$.

THEOREM 2.5 Under the same conditions of Theorem 2.2, suppose that $H_{i}$ are determined from (17) for some $\rho_{i}>0, i \in S$. Then, the matrix upper solution bound in (12) of Theorem 2.2 is independent of these $\rho_{i}$.

Proof: Given arbitrary but fixed $\rho_{i}>0$, we assume the upper bound as in (12). We consider now new values of $\rho_{i}$ in the form of $\widehat{\rho}_{i}=d_{i} \rho_{i}$ for arbitrary $d_{i}>0$. Set

$$
D=\left[\begin{array}{llll}
d_{1} & & & \\
& d_{2} & & \\
& & \ddots & \\
& & & d_{n}
\end{array}\right] .
$$


Let us denote quantities associated with $\widehat{\rho}_{i}$ by $\widehat{H}_{i}, \widehat{F}, \widehat{g}$, and $\widehat{\xi}$. Then, we see from (17) that $H_{i}$ become $\widehat{H}_{i}=d_{i} H_{i}$ for all $i$. Besides, by (8) and (9), $\widehat{F}$ and $\widehat{g}$ can be expressed as

$$
\widehat{F}=D^{-1} F D
$$

and

$$
\widehat{g}=D^{-1} g,
$$

respectively. Consequently, we obtain

$$
\widehat{\xi}=D^{-1} \xi .
$$

Hence,

$$
\widehat{\xi}_{i} \widehat{H}_{i}=\xi_{i} H_{i}, \forall i \in S .
$$

This implies that the upper bound in (12) is unchanged with the new values of $\rho_{i}$.

Theorem 2.5 allows us to use any $\rho_{i}>0$ in (17) while determining $H_{i}$.

To conclude this section, we give below two examples so as to illustrate the preceding results. We point out here that there are efficient algorithms, see $[2,11]$, for computing $H_{i}$ that satisfy (4) or, specifically, (17).

EXAMPLE 2.1 This example first appeared in $[8,22]$. It is revisited here to demonstrate that, with the new results in this work, we may get solution bounds tighter than or comparable to those in [22]. Let

$$
\begin{gathered}
A_{1}=\left[\begin{array}{rr}
1 & 1 \\
2 & -2
\end{array}\right], A_{2}=\left[\begin{array}{rr}
-3 & 2 \\
2 & -4
\end{array}\right], B_{1}=\left[\begin{array}{l}
1 \\
0
\end{array}\right], B_{2}=\left[\begin{array}{l}
2 \\
3
\end{array}\right], \\
\delta_{1,2}=\delta_{2,1}=0.1, Q_{1}=\left[\begin{array}{ll}
4 & 3 \\
3 & 4
\end{array}\right], \text { and } Q_{2}=\left[\begin{array}{ll}
6 & 2 \\
2 & 3
\end{array}\right] .
\end{gathered}
$$

Then, equation (1) has the solution

$$
P_{1}=\left[\begin{array}{ll}
4.4860 & 1.7725 \\
1.7725 & 1.1080
\end{array}\right] \text { and } P_{2}=\left[\begin{array}{ll}
0.6207 & 0.2185 \\
0.2185 & 0.2873
\end{array}\right] \text {. }
$$

Even though for each $i, B_{i} B_{i}^{T}$ is singular, condition (2) can still be satisfied on this example. It is known, see [22], that when $\alpha_{1}=3, \alpha_{2}=0.1, \beta_{i}=1$ and $\gamma_{i}=-1$ for $i=1,2$, the solution bounds are given by

$$
P_{1} \preceq\left[\begin{array}{rr}
12.5018 & 2.0902 \\
2.0902 & 4.4509
\end{array}\right], \quad P_{2} \preceq\left[\begin{array}{ll}
1.8045 & 0.1583 \\
0.1583 & 1.5730
\end{array}\right] .
$$


Now, using

$$
K_{1}=[2.6000,0.8950] \text { and } K_{2}=[0.5900,0.1467],
$$

we can easily verify that each $A_{i}-B_{i} K_{i}$ is stable. Moreover, based on (17) and Theorem 2.5, we choose $\rho_{1}=\rho_{2}=1$ to obtain

$$
H_{1}=\left[\begin{array}{ll}
0.5105 & 0.1584 \\
0.1584 & 0.2583
\end{array}\right] \text { and } H_{2}=\left[\begin{array}{ll}
0.1211 & 0.0273 \\
0.0273 & 0.1231
\end{array}\right]
$$

such that condition (4) holds.

Suppose next that $\beta_{i}=1$ and $\gamma_{i}=-1$ for $i=1,2$. We use Theorem 2.1 and (11) to find that

$$
F=\left[\begin{array}{rr}
1.0000 & -0.0222 \\
-0.0774 & 1.0000
\end{array}\right], g=\left[\begin{array}{c}
19.0378 \\
9.6941
\end{array}\right], \text { and } \xi=\left[\begin{array}{l}
19.2862 \\
11.1865
\end{array}\right] \text {. }
$$

Note here that condition (10) is met and hence $F$ is an $M$-matrix.

Finally, from Theorem 2.2, we arrive at the following matrix upper solution bound:

$$
P_{1} \preceq\left[\begin{array}{ll}
7.4634 & 1.8628 \\
1.8628 & 1.7131
\end{array}\right], P_{2} \preceq\left[\begin{array}{ll}
1.0739 & 0.3657 \\
0.3657 & 0.9246
\end{array}\right],
$$

which is tighter than the bound in (18).

EXAMPLE 2.2 Let

$$
\begin{gathered}
A_{1}=\left[\begin{array}{rr}
1 & 3 \\
0 & -7
\end{array}\right], A_{2}=\left[\begin{array}{rr}
2 & 2 \\
-1 & -4
\end{array}\right], B_{1}=\left[\begin{array}{l}
0 \\
2
\end{array}\right], B_{2}=\left[\begin{array}{l}
1 \\
3
\end{array}\right], \\
\delta_{1,2}=\delta_{2,1}=0.1, Q_{1}=\left[\begin{array}{ll}
19.4 & 17.9 \\
17.9 & 25.8
\end{array}\right], \text { and } Q_{2}=\left[\begin{array}{ll}
58.2 & 54.7 \\
54.7 & 60.8
\end{array}\right] .
\end{gathered}
$$

Then, equation (1) has the solution

$$
P_{1}=\left[\begin{array}{ll}
8 & 3 \\
3 & 2
\end{array}\right] \text { and } P_{2}=\left[\begin{array}{ll}
6 & 1 \\
1 & 2
\end{array}\right]
$$


However, condition (2) cannot be satisfied on this example because for each $i$,

$$
A_{i}^{T}+A_{i}-2 \alpha_{i} B_{i} B_{i}^{T}
$$

has an eigenvalue that remains positive for all $\alpha_{i}>0$.

Now, using

$$
K_{1}=[2,-1] \text { and } K_{2}=[2,1],
$$

we can easily verify that each $A_{i}-B_{i} K_{i}$ is stable. Moreover, based on (17) and Theorem 2.5, we choose $\rho_{1}=\rho_{2}=1$ to obtain

$$
H_{1}=\left[\begin{array}{ll}
0.8571 & 0.3393 \\
0.3393 & 0.3036
\end{array}\right] \text { and } H_{2}=\left[\begin{array}{ll}
1.0714 & 0.0714 \\
0.0714 & 0.0816
\end{array}\right]
$$

such that condition (4) holds.

Suppose next that $\beta_{i}=1$ and $\gamma_{i}=-1$ for $i=1,2$. We use Theorem 2.1 and (11) to find that

$$
F=\left[\begin{array}{rr}
1.0000 & -0.2812 \\
-0.1449 & 1.0000
\end{array}\right], g=\left[\begin{array}{c}
43.1932 \\
120.6624
\end{array}\right], \text { and } \xi=\left[\begin{array}{c}
80.3977 \\
132.3091
\end{array}\right] \text {. }
$$

Note here that condition (10) is met and hence $F$ is an $M$-matrix.

Finally, from Theorem 2.2, we arrive at the following matrix upper solution bound:

$$
P_{1} \preceq\left[\begin{array}{ll}
24.6008 & 13.7649 \\
13.7649 & 19.0537
\end{array}\right]=U_{1}^{(0)}, P_{2} \preceq\left[\begin{array}{rr}
16.9846 & 9.7188 \\
9.7188 & 10.6902
\end{array}\right]=U_{2}^{(0)},
$$

where the notation $U_{i}^{(0)}$ shall become clear in the next section.

\section{Iterative Refinement Algorithm}

In [23], an iterative refinement algorithm was investigated. It was shown to be able to noticeably sharpen the matrix upper solution bound in $[8,22]$ for the continuous coupled algebraic Riccati equation (1). We demonstrate here that a similar iterative refinement algorithm can further improve the matrix 
upper solution bound from Theorem 2.2.

Recall that the conditions of Theorem 2.2 imply condition (16).

THEOREM 3.1 Under the same assumptions as in Theorem 2.2, denote by $U_{i}^{(0)}$ the matrix upper bound in Theorem 2.2 and construct $\left\{U_{i}^{(k)}\right\}$ by

$$
\begin{aligned}
U_{i}^{(k)}= & \left(C_{i}+\gamma_{i} I\right)^{-T}\left[\left(C_{i}+\beta_{i} I\right)^{T} U_{i}^{(k-1)}\left(C_{i}+\beta_{i} I\right)-\left(\beta_{i}^{2}-\gamma_{i}^{2}\right) U_{i}^{(k-1)}\right. \\
& \left.+\left(\beta_{i}-\gamma_{i}\right)\left(\sum_{j \in S \backslash\{i\}} \delta_{i, j} U_{j}^{(k-1)}+Q_{i}+K_{i}^{T} K_{i}\right)\right]\left(C_{i}+\gamma_{i} I\right)^{-1}
\end{aligned}
$$

for $k=1,2, \ldots$ Then

$$
U_{i}^{(k)} \preceq U_{i}^{(k-1)}, \forall i \in S, k=0,1, \ldots
$$

In particular, the above $\preceq$ can be replaced with $\prec$ when condition (16) holds with $\prec$.

Proof: First of all, we claim that $U_{i}^{(0)}$, the matrix upper bound in Theorem 2.2 , satisfies

$$
U_{i}^{(0)} \preceq \xi_{i} H_{i}, \forall i \in S
$$

This is because

$$
\begin{aligned}
U_{i}^{(0)}-\xi_{i} H_{i} \\
=\left(C_{i}+\gamma_{i} I\right)^{-T}\left[\xi_{i}\left(C_{i}+\beta_{i} I\right)^{T} H_{i}\left(C_{i}+\beta_{i} I\right)-\xi_{i}\left(C_{i}+\gamma_{i} I\right)^{T} H_{i}\left(C_{i}+\gamma_{i} I\right)\right. \\
\left.\quad-\left(\beta_{i}^{2}-\gamma_{i}^{2}\right) \xi_{i} H_{i}+\left(\beta_{i}-\gamma_{i}\right)\left(\sum_{j \in S \backslash\{i\}} \delta_{i, j} \xi_{j} H_{j}+Q_{i}+K_{i}^{T} K_{i}\right)\right]\left(C_{i}+\gamma_{i} I\right)^{-1} \\
=\left(C_{i}+\gamma_{i} I\right)^{-T}\left[\xi_{i}\left(C_{i}^{T} H_{i}+H_{i} C_{i}\right)+\sum_{j \in S \backslash\{i\}} \delta_{i, j} \xi_{j} H_{j}+Q_{i}+K_{i}^{T} K_{i}\right]\left(C_{i}+\gamma_{i} I\right)^{-1} \\
\preceq 0
\end{aligned}
$$

in light of condition (16). It is also obvious that if (16) holds with $\prec$, then $U_{i} \prec \xi_{i} H_{i}$. 
We proceed now by induction. Observe that

$$
\begin{aligned}
& U_{i}^{(1)}-U_{i}^{(0)} \\
& =\left(C_{i}+\gamma_{i} I\right)^{-T}\left[\left(C_{i}+\beta_{i} I\right)^{T}\left(U_{i}^{(0)}-\xi_{i} H_{i}\right)\left(C_{i}+\beta_{i} I\right)-\left(\beta_{i}^{2}-\gamma_{i}^{2}\right)\left(U_{i}^{(0)}-\xi_{i} H_{i}\right)\right. \\
& \left.\quad+\left(\beta_{i}-\gamma_{i}\right) \sum_{j \in S \backslash\{i\}} \delta_{i, j}\left(U_{j}^{(0)}-\xi_{j} H_{j}\right)\right]\left(C_{i}+\gamma_{i} I\right)^{-1} \preceq 0 .
\end{aligned}
$$

In particular, if condition (16) is in the stronger $\prec$ form, then the above $\preceq$ can be replaced with $\prec$ too.

Next, we suppose that $U_{i}^{(l+1)} \preceq U_{i}^{(l)}$ for all $i \in S$ and $l=0, \ldots, k-1$, with $\prec$ here whenever we have $\prec$ in condition (16). Then

$$
\begin{aligned}
& U_{i}^{(k+1)}-U_{i}^{(k)} \\
& =\left(C_{i}+\gamma_{i} I\right)^{-T}\left[\left(C_{i}+\beta_{i} I\right)^{T}\left(U_{i}^{(k)}-U_{i}^{(k-1)}\right)\left(C_{i}+\beta_{i} I\right)-\left(\beta_{i}^{2}-\gamma_{i}^{2}\right)\left(U_{i}^{(k)}-U_{i}^{(k-1)}\right)\right. \\
& \left.\quad+\left(\beta_{i}-\gamma_{i}\right) \sum_{j \in S \backslash\{i\}} \delta_{i, j}\left(U_{j}^{(k)}-U_{j}^{(k-1)}\right)\right]\left(C_{i}+\gamma_{i} I\right)^{-1} \preceq 0 .
\end{aligned}
$$

This completes the proof.

Theorem 3.1 establishes an iterative algorithm for improving the matrix upper solution bound in Theorem 2.2. We also point out that [23, Algorithm 4.1] is now a special case, i.e. $K_{i}=\alpha_{i} B_{i}^{T}$, of Theorem 3.1. Moreover, compared with [23, Algorithm 4.1], Theorem 3.1 holds under the less restrictive stabilizability condition.

To illustrate the iterative refinement algorithm in Theorem 3.1, we consider Example 2.2 as in Section 2.

EXAMPLE 3.1 Using the same settings as in Example 2.2, we apply Theorem 3.1 with 5 iterations, starting with $U_{i}^{(0)}$ as given in (19), to obtain an improved matrix upper solution bound as follows:

$$
U_{1}^{(5)}=\left[\begin{array}{rr}
10.2064 & 5.6417 \\
5.6417 & 6.1333
\end{array}\right] \text { and } U_{2}^{(5)}=\left[\begin{array}{rr}
10.3834 & 4.7746 \\
4.7746 & 5.3169
\end{array}\right]
$$

Clearly, this result is much tighter compared with $U_{i}^{(0)}$ in (19). 


\section{Concluding Remarks}

In this paper, we generalize existing matrix upper solution bounds in $[8,9$, $22,23]$ within a broader framework involving the continuous coupled algebraic Riccati equation. Our results here remain applicable even in presence of rank deficiency involving the control matrices. They also include the existing results in $[8,9,22,23]$ as special cases.

Following [22, 23], we approach the problem of bounding the solution of the continuous coupled algebraic Riccati equation in a unifying yet flexible manner by using parameters such as $\beta_{i}$ and $\gamma_{i}$. It provides a useful vantage point in search of tighter solution bounds. We are able to show, for example, that [9, Theorem 2.2] always produces better bounds than [9, Theorem 2.1]. At the same time, it also raises an interesting question as to how quantities such as $\gamma_{i}, K_{i}$, and $H_{i}$ would affect the tightness of resulting bounds. Even though our Theorem 2.5 confirms that $H_{i}$ may be determined by (17) with arbitrary $\rho_{i}>0$, other choices of $H_{i}$ deserve further investigation too.

An important aspect of the research on the continuous coupled algebraic Riccati equation is to seek tighter and also simpler matrix solution bounds. Much work is still needed in this regard. We comment that (20) could serve as a slightly simpler upper solution bound in a less demanding situation.

It is also worth mentioning that our results could have implications in the numerical solution of the continuous coupled algebraic Riccati equation. In particular, we are concerned with the utilization of certain solution bounds as refined initial approximations for an iterative solution algorithm. It can be shown, for example, that $B_{i}^{T} U_{i}^{(k)}$, where $U_{i}^{(k)}$ is given as in Theorem 3.1, stabilizes $\left(A_{i}, B_{i}\right)$ for all $i$, which is important for the convergence of some iterative solution algorithms; see, for instance, $[12,16]$. Such algorithms are part of our ongoing investigation on coupled algebraic Riccati equations.

Another possible extension of our work here is to apply a similar method-

ology to the sensitivity analysis of the continuous coupled algebraic Riccati equation, i.e. to bounding the perturbations in the solution subject to variations in system parameters.

In the meantime, as pointed out by the reviewers, there are several other 
interesting questions to explore in our future study. Firstly, our results may be further improved in light of the conditions in $[1,5,12]$ for the existence of the positive semidefinite solution $P_{i}$ of the continuous coupled algebraic Riccati equation in (1), which would have a more practical interpretation in terms of the dynamics of the Markovian jump linear system. Secondly, our methodology may be extended to the so-called semi-Markovian jump linear system, see [18, 19], which turns out to be more applicable than the conventional Markovian jump linear system. And lastly, but certainly not leastly, our approach here may be generalized in the same spirit as [15] to deal with continuous coupled algebraic Riccati equations involving singularities.

Acknowledgements. The authors would like to thank the anonymous reviewers for their constructive comments that have helped improve the presentation of this paper.

\section{References}

[1] H. Abou-Kandil, G. Freiling, and G. Jank, Solution and asymptotic behavior of coupled Riccati equations in jump linear systems, IEEE Transactions on Automatic Control, 39: 1631-1636, 1994.

[2] R. Bartels and G. Stewart, Algorithm 432: Solution of the matrix equation $A X+X B=C$, Communications of the ACM, 15: 820-826, 1972.

[3] D. Bernstein, Matrix Mathematics: Theory, Facts, and Formulas, 2nd edition, Princeton University Press, 2005.

[4] A. Berman and R. Plemmons, Nonnegative Matrices in the Mathematical Sciences, SIAM, 1994.

[5] A. Czornik and A. Nawrat, On the bounds on the solutions of the algebraic Lyapunov and Riccati equations, Archives of Control Sciences, 10: 197-244, 2000.

[6] A. Czornik and A. Swierniak, Lower bounds on the solution of coupled algebraic Riccati equation, Automatica, 37: 619-624, 2001.

[7] A. Czornik and A. Swierniak, Upper bounds on the solution of coupled algebraic Riccati equation, Journal of Inequalities and Applications, 6: 373-385, 2001. 
[8] R. Davies, P. Shi, and R. Wiltshire, Upper solution bounds of the continuous and discrete coupled algebraic Riccati equations, Automatica, 44: 1088-1096, 2008.

[9] R. Davies, P. Shi, and R. Wiltshire, New upper matrix bounds for the solution of the continuous algebraic Riccati matrix equation, International Journal of Control, Automation, and Systems, 6: 776-784, 2008.

[10] L. Gao, A. Xue, and Y. Sun, Matrix bounds for the coupled algebraic Riccati equation, Proceedings of the Fourth World Congress on Intelligent Control and Automation, 180-183, 2002.

[11] G. Golub, S. Nash, and C. Van Loan, A Hessenberg-Schur method for the problem $A X+X B=C$, IEEE Transactions on Automatic Control, 24: 909-913, 1979.

[12] C. Guo, Iterative methods for a linear perturbed algebraic matrix Riccati equation arising in stochastic control, Numerical Functional Analysis and Optimization, 34: 516-529, 2013.

[13] R. Horn and C. Johnson, Matrix Analysis, Cambridge University Press, 1990.

[14] R. Horn and C. Johnson, Topics in Matrix Analysis, Cambridge University Press, 1995.

[15] C. Huang, J. Tsai, S. Guo, Y. Sun, and L. Shieh, Solving algebraic Riccati equation for singular system based on matrix sign function, International Journal of Innovative Computing, Information and Control, 9: 2771-2788, 2013.

[16] I. Ivanov, On some iterations for optimal control of jump linear equations, Nonlinear Analysis, 69: 4012-4024, 2008.

[17] P. Lancaster and L. Rodman, Algebraic Riccati Equations, Oxford University Press, 1995.

[18] F. Li, P. Shi, L. Wu, M. Basin, and C. Lim, Quantized control design for cognitive radio networks modeled as nonlinear semiMarkovian jump systems, IEEE Transactions on Industrial Electronics, DOI: 10.1109/TIE.2014.2351379. 
[19] F. Li, L. Wu, P. Shi, and C. Lim, State estimation and sliding mode control for semi-Markovian jump systems with mismatched uncertainties, Automatica, DOI: 10.1016/j.automatica.2014.10.065.

[20] M. Mariton, Jump Linear Systems in Automatic Control, Marcel Dekker, New York, 1990.

[21] V. Sima, An efficient Schur method to solve the stabilizing problem, IEEE Transactions on Automatic Control, 26: 724-725, 1981.

[22] J. Xu, Unified, improved matrix upper bound on the solution of the continuous coupled algebraic Riccati equation, Journal of the Franklin Institute, 350: 1634-1648, 2013.

[23] J. Xu and M. Xiao, On the iterative refinement of matrix upper bounds for the solution of continuous coupled algebraic Riccati equations, Automatica, 49: 2168-2175, 2013.

[24] K. Yasuda and K. Hirai, Upper and lower bounds on the solution of the algebraic Riccati equation, IEEE Transactions on Automatic Control, 24: 483-487, 1979. 